\title{
Follow-up colonoscopy after an abnormal stool-based colorectal cancer screening result: analysis of steps in the colonoscopy completion process
}

Gloria D. Coronado ${ }^{1 *}$, Alexandra Kihn-Stang ${ }^{1,2}$, Matthew T. Slaughter ${ }^{1}$, Amanda F. Petrik1, Jamie H. Thompson ${ }^{1}$, Jennifer S. Rivelli ${ }^{1}$, Ricardo Jimenez ${ }^{3}$, Jeffrey Gibbs ${ }^{3}$, Neha Yadav ${ }^{3}$ and Rajasekhara R. Mummadi4

\begin{abstract}
Background: Delays in receiving follow-up colonoscopy after an abnormal fecal immunochemical test (FIT) result are associated with increased colorectal cancer incidence and mortality. Little is known about patterns of follow-up colonoscopy completion in federally qualified health centers.

Methods: We abstracted the medical records of health center patients, aged 50-75 years, who had an abnormal FIT result between August 5, 2017 and August 4, $2018(N=711)$. We assessed one-year rates of colonoscopy referral, pre-procedure visit completion, colonoscopy completion, and time to colonoscopy; associations between these outcomes and patient characteristics; and reasons for non-completion found in the medical record.
\end{abstract}

Results: Of the 711 patients with an abnormal FIT result, $90 \%$ were referred to colonoscopy, but only 52\% completed a pre-procedure visit, and $43 \%$ completed a colonoscopy within 1 year. Median time to colonoscopy was 83 days (interquartile range: 52-131 days). Pre-procedure visit and colonoscopy completion rates were relatively low in patients aged 65-75 (vs. 50-64), who were uninsured (vs. insured) or had no clinic visit in the prior year (vs. $\geq 1$ clinic visit). Common reasons listed for non-completion were that the patient declined, or the provider could not reach the patient.

Discussion: Efforts to improve follow-up colonoscopy rates in health centers might focus on supporting the care transition from primary to specialty gastroenterology care and emphasize care for older uninsured patients and those having no recent clinic visits. Our findings can inform efforts to improve follow-up colonoscopy uptake, reduce time to colonoscopy receipt, and save lives from colorectal cancer.

Trial registration: National Clinical Trial (NCT) Identifier: NCT03925883.

Keywords: Follow-up colonoscopy, Time to colonoscopy, Reasons for non-adherence, Colonoscopy pathway, Federally qualified health centers

*Correspondence: Gloria.D.Coronado@kpchr.org

${ }^{1}$ Center for Health Research, Kaiser Permanente Northwest, 3800 North Interstate Avenue, Portland, OR 97227, USA

Full list of author information is available at the end of the article

\begin{abstract}
Background
Colorectal cancer $(\mathrm{CRC})$ is the third leading cause of cancer death in the United States, accounting for an estimated 51,000 deaths in 2019 [1]. Due to increased screening, CRC incidence and mortality has declined considerably over the past four decades, however many
\end{abstract}


people who should be screened still do not get screened [2]. Fecal immunochemical tests (FITs) are a low-cost alternative to colonoscopy for identifying patients at risk of CRC, however, for FITs to be effective, patients who receive an abnormal FIT result must obtain a timely follow-up colonoscopy in order to remove precancerous polyps or find cancer in early forms that can be successfully treated. Troublingly, follow-up colonoscopy rates are low in most US healthcare settings and are particularly low in federally qualified health centers (FQHCs), where published rates range from 18 to $57 \%$ [3-7]. These rates are well below the $80 \%$ target set by the Multi-Society Task Force on Colorectal Cancer [8]. Compared with health care settings that have integrated specialty care services, FQHCs face unique challenges in coordinating care with specialty colonoscopy providers located in separate health care systems.

Delays in receiving follow-up colonoscopy are associated with increased CRC incidence and mortality. Lee and colleagues reported a $31 \%$ higher risk of CRC and a two-fold higher risk for advanced stage disease among adults who delayed colonoscopy by 6 months or more compared to those who obtained a colonoscopy within 1-3 months of an abnormal FIT [9]. Similar findings were reported in a Kaiser Permanente study, where a 10 -month delay was associated with a $48 \%$ increase in CRC risk [10]. Meester and colleagues used modeling to estimate a $4 \%$ increase in CRC incidence and $16 \%$ increase in mortality among adults who delayed followup colonoscopy by 12 months versus those who received it within 2 weeks [11].

For many patients, obtaining a follow-up colonoscopy can be a complex process, often requiring the completion of several steps, including obtaining a referral to a gastroenterology (GI) practice; possibly attending a pre-procedure visit; arranging an escort and transportation to and from the procedure; completing bowel preparation; and attending the colonoscopy procedure. Each of these steps can create compounding barriers to colonoscopy completion. Analyses of qualitative interviews with patients have identified several barriers, including lack of knowledge about the need for colonoscopy, difficulty arranging transportation to and from the procedure, concerns about cost, inability to leave work to attend the procedure, fear of discomfort, and difficulty completing bowel preparation [12-14].

In a previous retrospective cohort study conducted in a San Francisco-based integrated safety net health system, Issaka and colleagues reported that among patients with an abnormal FIT result, $87 \%$ obtained a colonoscopy referral, $65 \%$ attended a pre-procedure visit, and $56 \%$ obtained a colonoscopy [6]. Notably, patients in this study were referred for colonoscopy to a single integrated hospital system, and little is known about patterns of participation across these colonoscopy completion steps in non-integrated health centers.

As part of the Predicting and Addressing Colonoscopy Non-adherence in Community Settings (PRECISE) study, we used data abstracted from the medical records of patients with an abnormal FIT result to explore patterns of follow-up colonoscopy completion and identify key steps in the process at which patients stopped seeking care (referral, pre-procedure visit, or colonoscopy completion) and reasons for this discontinuation. Our analysis builds on prior research by assessing patient characteristics associated with successful completion of intermediate steps, and reasons listed in the electronic health record (EHR) for discontinuation of each step. Moreover, our study was set in a non-integrated health center and our analysis includes more abstracted health records than prior research [6].

\section{Methods}

The PRECISE study is an individual randomized controlled trial of patient navigation versus usual care for follow-up colonoscopy completion among patients who receive an abnormal FIT result at Sea Mar Community Health Centers [15]. As part of PRECISE, we conducted a baseline assessment of follow-up colonoscopy in a retrospective cohort of patients who had an abnormal FIT result over a one-year interval from August 5, 2017, to August 4, 2018. We abstracted the health records of these patients to assess completion of intermediate steps in obtaining a follow-up colonoscopy (i.e., referral to GI, completion of pre-procedure visit, and completion of colonoscopy procedure) and time to colonoscopy in the 365 days following the abnormal FIT result. The PRECISE study protocol, including this assessment, was reviewed, and approved by the Institutional Review Board at Kaiser Permanente Northwest [protocol \# STUDY00000779]. We obtained a waiver of informed consent, given the minimal risks posed to patients. Our study adheres to CONSORT reporting requirements for clinical studies.

\section{Setting and study population}

Sea Mar Community Health Centers is a large FQHC that operates 32 primary care clinics in western Washington State. Sea Mar provides a broad range of health and human services to a large and diverse population of more than 305,000 individuals across 13 counties, $37 \%$ of whom are Latinx. Annually, about 29,000 Sea Mar patients are age-eligible for FIT screening. In 2018, the Sea Mar CRC screening rate was $45 \%$, and the average FIT positivity rate was $9 \%$; thus, about 700 patients received an abnormal FIT test result. Sea Mar refers patients with abnormal FIT results to one of several 
community GI practices. In 2018, the Sea Mar quality improvement manager requested records from the GI practices of all patients who had had an abnormal FIT result and a GI referral, but no evidence of a completed colonoscopy; these records were in the EHR and included in our analyses. Despite these efforts, we cannot be sure that all records of completed colonoscopies were in the EHR.

\section{Study procedures}

The Sea Mar EHR analyst identified all patients aged 50-75 years with an abnormal FIT result between August 5, 2017 and August 4, $2018(\mathrm{~N}=711)$. Chart abstraction was performed after August 4, 2018, allowing at least one year of follow-up time between the abnormal FIT result and chart abstraction. No exclusions were applied.

A study team member (AFP) created chart abstraction forms in REDCap (Nashville, TN), based on forms used in previous research [5]. The forms were reviewed by the Principal Investigator (GDC) and pilot-tested by the study analyst and a chart abstractor (MS, AKS). Chart abstraction was carried out by two abstractors, a research team member and a clinic staff member. All chart abstraction was completed on-site at a Sea Mar clinic, using the EHR (Allscripts, Chicago, Illinois). Chart abstractors reviewed scanned documents, provider orders, free text fields, and any other data not captured using automated queries. Sensitivity analyses were not completed as we assumed that missing data were truly missing and treated the chart review as the reference standard.

\section{Study outcomes}

Outcomes included rates of referral to colonoscopy, completion of a pre-procedure visit, completion of a follow-up colonoscopy procedure, and time to followup colonoscopy completion. Pre-procedure visit reports were present in 242 of the 711 charts. Because we had no way of knowing whether a pre-procedure visit had been recommended by the GI practice, for the purposes of statistical analysis, we counted all patients with a completed colonoscopy as having completed a pre-procedure visit. This resulted in an additional 123 patients classified as having a pre-procedure visit. We report associations between patient demographic and clinical characteristics and completion of the pre-procedure visit and the colonoscopy. When health record documentation provided reasons that steps were not completed, we recorded that information for analysis.

\section{Statistical analysis}

We report frequencies of socio-demographic characteristics of our study sample. Among the 642 patients who received a referral, we calculated the proportions who completed (1) a pre-procedure visit and (2) a follow-up colonoscopy overall and by referring GI practice. For the 309 patients who completed a colonoscopy, we also calculated median time and interquartile range for colonoscopy completion overall and by GI practice. We used multivariate logistic regression to calculate the association between patient characteristics and completion of a pre-procedure visit and of colonoscopy. Patient characteristics included age, sex, race, ethnicity, preferred language, marital status, insurance type, and number of clinic visits in the year prior to the abnormal FIT. We report adjusted odds ratios and 95\% confidence intervals. We also report the concordance between EHR codes and chart abstraction in rates of referral and colonoscopy completion using EHR codes, and rates obtained during chart abstraction. All analyses were performed using SAS version 9.4 (Cary, NC).

\section{Results}

A total of 711 patients had an abnormal FIT result during the 365-day catchment interval and were included in analyses (Table 1). Patients had a mean age of 61 and were predominantly non-Hispanic white $(83 \%)$; $81 \%$ preferred speaking English. Ninety-one percent had health insurance, and $72 \%$ had at least one clinic visit in the year prior to their abnormal FIT result.

Compared to patients aged 50-64 years, patients aged 65 years and older had lower odds of completing a pre-procedure visit (OR: 0.43 ; 95\% CI $0.25,0.72$ ) and of completing a colonoscopy (OR: 0.46; $95 \%$ CI: $0.27,0.79$ ). Insurance status was associated with pre-procedure visit completion: patients with commercial insurance or Medicare had significantly higher odds of pre-procedure visit completion than patients who were uninsured (commercial insurance OR: 2.22; 95\% CI: 1.08, 4.57; Medicare OR: 4.19; 95\% CI: 1.83, 9.58); rates were also higher for Medicaid-enrolled patients, but the difference did not reach significance (OR: 1.96; 95\% CI: 0.98, 3.92). Medicare-enrolled patients also had a higher-odds of completing a colonoscopy than uninsured patients (OR: 2.96; 95\% CI: $1.29,6.80)$. Having a clinic visit in the year prior to the abnormal FIT result was associated with higher odds of pre-procedure and colonoscopy completion, with the strength of these associations increasing with increasing numbers of visits. Neither sex, race, ethnicity, language, or marital status were significantly associated with completion of a pre-procedure visit or a colonoscopy procedure.

When we compared the concordance of data obtained from automated EHR queries and chart abstraction, we observed high concordance between the two data sources on colonoscopy referral (92\%) and colonoscopy 
Table 1 Characteristics of patients with abnormal FIT result $(N=711)$

\begin{tabular}{|c|c|c|c|c|c|c|c|c|}
\hline \multirow[t]{2}{*}{ Characteristic } & \multicolumn{2}{|c|}{$\begin{array}{l}\text { Total sample } \\
(\mathrm{N}=711)\end{array}$} & \multicolumn{3}{|c|}{$\begin{array}{l}\text { Completed pre-procedure visit } \\
(\mathrm{N}=365)\end{array}$} & \multicolumn{3}{|c|}{$\begin{array}{l}\text { Completed colonoscopy } \\
(\mathrm{N}=309)\end{array}$} \\
\hline & $\mathrm{N}$ & $\%$ & $\mathrm{~N}$ & $\%^{\mathrm{a}}$ & OR $(95 \% \mathrm{Cl})$ & $\mathrm{N}$ & $\%$ & OR $(95 \% \mathrm{Cl})$ \\
\hline Age (mean, SD) & \multicolumn{8}{|c|}{$61.3(6.4)$} \\
\hline $50-64$ & 486 & 68.4 & 262 & 53.9 & Ref & 224 & 46.1 & Ref \\
\hline $65-75$ & 225 & 31.7 & 103 & 45.8 & $.43(.25-.72)$ & 85 & 37.8 & $.46(.27-.79)$ \\
\hline \multicolumn{9}{|l|}{ Sex } \\
\hline Male & 391 & 55.0 & 197 & 50.4 & Ref & 169 & 43.2 & Ref \\
\hline Female & 320 & 45.0 & 168 & 52.5 & $1.09(.79-1.50)$ & 140 & 43.8 & $1.05(.76-1.44)$ \\
\hline \multicolumn{9}{|l|}{ Race } \\
\hline White & 529 & 74.4 & 269 & 50.9 & Ref & 229 & 43.3 & Ref \\
\hline Asian & 35 & 4.9 & 17 & 48.6 & $.93(.41-2.10)$ & 13 & 37.1 & $.71(.31-1.63)$ \\
\hline Black/African American & 31 & 4.4 & 18 & 58.1 & $1.77(.81-3.86)$ & 17 & 54.8 & $1.97(.91-4.24)$ \\
\hline Other/more than 1 race & 90 & 12.7 & 48 & 53.3 & $.86(.51-1.46)$ & 38 & 42.22 & $.75(.44-1.29)$ \\
\hline Not reported & 26 & 3.7 & 13 & 50.0 & - & 12 & 46.2 & - \\
\hline \multicolumn{9}{|l|}{ Ethnicity $^{b}$} \\
\hline Hispanic/Latino & 121 & 17.0 & 74 & 61.2 & $1.56(.79-3.10)$ & 62 & 51.2 & $1.26(.64-2.47)$ \\
\hline Non-Hispanic/Latino & 588 & 82.7 & 290 & 49.3 & Ref & 246 & 41.8 & Ref \\
\hline \multicolumn{9}{|l|}{ Language } \\
\hline English & 575 & 80.9 & 281 & 48.9 & Ref & 238 & 41.4 & Ref \\
\hline Non-English & 136 & 19.1 & 84 & 61.8 & $1.67(.90-3.13)$ & 71 & 52.2 & $1.71(.92-3.18)$ \\
\hline \multicolumn{9}{|l|}{ Marital status } \\
\hline Single & 356 & 50.1 & 179 & 50.3 & Ref & 153 & 43.0 & Ref \\
\hline Married & 222 & 31.2 & 114 & 51.4 & $1.03(.70-1.51)$ & 97 & 43.7 & $1.03(.71-1.51)$ \\
\hline Separated/divorced/widowed & 133 & 18.7 & 72 & 54.1 & $1.26(.82-1.92)$ & 59 & 44.4 & $1.15(.75-1.76)$ \\
\hline \multicolumn{9}{|l|}{ Insurance } \\
\hline Medicare & 135 & 19.0 & 72 & 53.3 & $4.19(1.83-9.58)$ & 59 & 43.7 & $2.96(1.29-6.80)$ \\
\hline Commercial & 131 & 18.4 & 67 & 51.2 & $2.22(1.08-4.57)$ & 58 & 44.3 & $1.91(.93-3.93)$ \\
\hline Medicaid & 380 & 53.5 & 198 & 52.1 & $1.96(.98-3.92)$ & 167 & 44.0 & $1.57(.79-3.15)$ \\
\hline Uninsured & 65 & 9.1 & 28 & 43.1 & Ref & 25 & 38.5 & Ref \\
\hline \multicolumn{9}{|c|}{ Clinic visits in year prior to Index FIT result ${ }^{c}$} \\
\hline 0 & 203 & 28.6 & 77 & 37.9 & Ref & 62 & 30.5 & Ref \\
\hline 1 & 100 & 14.1 & 52 & 52.0 & $1.72(1.04-2.86)$ & 44 & 44.0 & $1.73(1.03-2.90)$ \\
\hline $2-3$ & 185 & 26.0 & 98 & 53.0 & $1.82(1.19-2.78)$ & 87 & 47.0 & $1.94(1.29-3.08)$ \\
\hline $4+$ & 223 & 31.4 & 138 & 61.9 & $2.53(1.67-3.82)$ & 116 & 52.0 & $2.38(1.56-3.62)$ \\
\hline
\end{tabular}

Bolded text indicates statistical significance

${ }^{\text {a }}$ All patients with completed colonoscopy were assumed to have completed a pre-procedure visit

${ }^{b} 2$ patients did not report ethnicity, percentages may not total $100 \%$

${ }^{\mathrm{c}}$ Mean clinic visits in past year $=2.72$ (standard deviation $=2.91$ )

completion (96\%). Automated EHR query data showed that of the 711 patients with an abnormal FIT, 83\% were referred to GI, and $42 \%$ completed a colonoscopy. In chart-abstracted data, we found evidence that $90 \%$ were referred to GI and 43\% completed a colonoscopy.

While $90 \%$ of patients with abnormal FIT were referred to a GI clinic, only $52 \%$ attended a pre-procedure visit and $43 \%$ completed a colonoscopy within the year following their abnormal FIT result (Fig. 1). Rates of follow-up colonoscopy completion ranged from 41 to $55 \%$ across the 10 most frequently referred GI practices (Table 2). The proportion of patients who completed a colonoscopy over time is shown in Fig. 2. Among the 309 patients who completed a colonoscopy, median time to completion was 83.0 days, ranging from 75.0 days to 112.5 days across the $10 \mathrm{GI}$ practices.

Among the 711 patients who had an abnormal FIT, 402 (57\%) did not complete a colonoscopy; 69 (10\% of the 711) received no referral, 277 (39\%) received a referral but did not complete a pre-procedure visit, and $56(8 \%)$ 


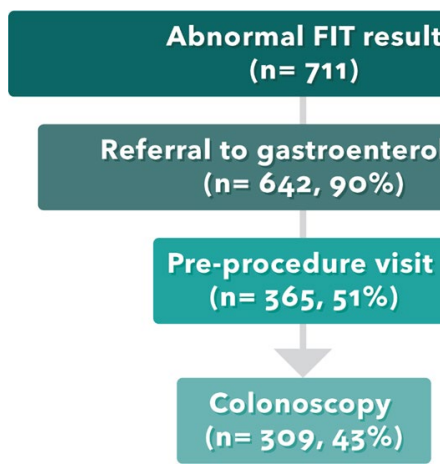

Fig. 1 Number and proportion of patients that received each step in the colonoscopy completion process within 12 months of an abnormal fecal immunochemical test (FIT) result. Of 711 patients who received an abnormal FIT result, 90\% were referred to gastroenterology, $51 \%$ attended a pre-procedure visit with a gastroenterologist, and $43 \%$ completed a colonoscopy within 12 months.

completed a pre-procedure visit but did not complete a colonoscopy. A reason for not completing the colonoscopy was present in 246 charts (61\% of those who did not complete a colonoscopy); reasons provided are summarized in Table 3. The most common reasons were that the patient declined, missed, or canceled an appointment (19\%) or the provider was unable to reach the patient (15\%).

\section{Discussion}

Using medical record abstraction, we assessed patterns of follow-up colonoscopy completion among patients of a large, diverse FQHC in Western Washington who received an abnormal FIT result. While $90 \%$ of these patients were referred to colonoscopy, only $52 \%$ attended a pre-procedure visit and $43 \%$ completed a colonoscopy within the year following their abnormal FIT result, well below the national benchmark of $80 \%$ [8]. Attrition was highest in the intermediate step between the GI referral and the pre-procedure visit (38\% percentage point drop). Efforts to improve follow-up colonoscopy rates in $\mathrm{FQHCs}$ might focus on supporting the care transition from primary care to specialty gastroenterology care. This may be particularly needed for patients aged $65-75$, those without a clinic visit in the prior year, and those who lack health insurance.

Given that adults with an abnormal FIT result have a 4-9\% chance of having undiagnosed colorectal cancer and a 2.4-fold elevated risk of dying from the disease than those with normal FIT results [8, 16-19], efforts are needed to improve follow-up colonoscopy rates in these settings. Prior studies have documented six-month FQHC follow-up colonoscopy completion rates between 18 and $57 \%$, and our rate of $37 \%$ was within that range [3, $4,20]$. Our observed $43 \%$ one-year colonoscopy completion rate was lower than that found in three prior studies in similar settings (53-58\%): our team previously reported a $53 \%$ one-year follow-up colonoscopy rate in a sample of 2,018 patients from eight non-integrated FQHCs [21], and two other studies, Issaka and colleagues'

Table 2 Colonoscopy completion by gastroenterology facility ( $n=642$ referred adults)

\begin{tabular}{|c|c|c|c|c|c|c|c|}
\hline \multirow{2}{*}{$\begin{array}{l}\text { Gastroenterology facility }{ }^{a} \\
\text { Overall }\end{array}$} & \multirow{2}{*}{$\begin{array}{l}\text { Referred } \\
\mathbf{N} \\
642\end{array}$} & \multicolumn{2}{|c|}{$\begin{array}{l}\text { Completed pre-procedure } \\
\text { visit } \\
\mathrm{N}(\%)^{\mathrm{b}}\end{array}$} & \multicolumn{2}{|c|}{$\begin{array}{l}\text { Colonoscopy completed } \\
\mathrm{N}(\%)^{\mathrm{b}}\end{array}$} & \multicolumn{2}{|c|}{$\begin{array}{l}\text { Time to colonoscopyc } \\
\text { Median days (IQR) }\end{array}$} \\
\hline & & 365 & $(56.9)$ & 305 & $(43.5)$ & 83.0 & $(52-131)$ \\
\hline Facility 1 & 94 & 52 & $(55.3)$ & 49 & $(52.1)$ & 90.0 & $(44-125)$ \\
\hline Facility 2 & 31 & 21 & $(67.7)$ & 16 & (51.6) & 75.0 & $(49.5-119.5)$ \\
\hline Facility 3 & 56 & 26 & $(46.4)$ & 24 & $(42.9)$ & 84.5 & $(57.5-162)$ \\
\hline Facility 4 & 53 & 34 & $(64.2)$ & 25 & $(47.2)$ & 90.0 & $(68-133)$ \\
\hline Facility 5 & 26 & 13 & $(50.0)$ & 12 & $(46.2)$ & 87.0 & $(68-129.5)$ \\
\hline Facility 6 & 57 & 34 & $(59.7)$ & 27 & $(47.4)$ & 79.0 & $(39-122)$ \\
\hline Facility 7 & 36 & 21 & $(58.3)$ & 16 & $(44.4)$ & 76.5 & $(64.5-155.5)$ \\
\hline Facility 8 & 40 & 27 & $(67.5)$ & 22 & $(55.0)$ & 112.5 & $(73-144)$ \\
\hline Facility 9 & 73 & 31 & $(42.5)$ & 30 & $(41.1)$ & 85.5 & $(61-133)$ \\
\hline Facility 10 & 69 & 44 & $(63.8)$ & 37 & (53.6) & 86.0 & $(51-115)$ \\
\hline Other/Unknown & 96 & 62 & (57.9) & 51 & $(47.7)$ & 70.0 & $(45-138)$ \\
\hline
\end{tabular}

${ }^{\mathrm{a}}$ Initial referral location, patients may have transferred care between referral and pre-procedure visit/colonoscopy. Unknown facilities have been excluded from this table; percentages may not total 100

${ }^{b}$ Percentages are based on the number referred for a given practice

' Median time among those who completed a colonoscopy 


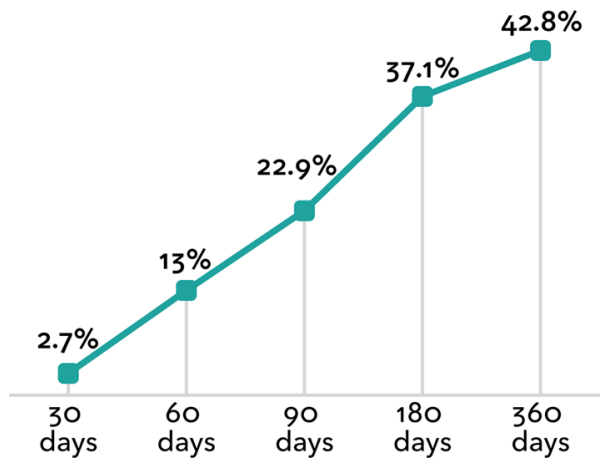

Fig. 2 Time from abnormal fecal immunochemical test (FIT) result to colonoscopy receipt, among 711 patients with an abnormal FIT result. Among patients with an abnormal FIT result, 22.9\% completed a colonoscopy within 90 days, $37.1 \%$ completed a colonoscopy within 180 days, and $42.8 \%$ completed a colonoscopy within 360 days

study in San Francisco and one in Dallas, found one-year completion rates of $56 \%$ and $58 \%$, respectively $[6,20]$. However, patients in the San Francisco and Dallas studies were served by integrated safety-net systems that generally referred to a single network hospital, which likely facilitated both colonoscopy completion and data capture $[6,20]$.

The median time to follow-up colonoscopy that we observed, 83 days, was within the range reported in prior studies (64 days to 184 days) [4-6, 20]. Our observed time to colonoscopy was within the 90 -day benchmark set by the PROSPR initiative [22], suggesting that patients who successfully access GI services generally are able to obtain a timely procedure. Notably, we observed a 37.5day variation in median time to follow-up colonoscopy across GI referral sites, (range $=75$ to 112.5 days).
Nearly $40 \%$ of patients with no follow-up colonoscopy had no reason documented in their medical record. Among those for whom a reason was documented, the most common reasons were that the patient declined, missed, or canceled an appointment, or could not be reached. These findings were similar to those of prior research by our team showing that common reasons were a patient's declination or appointment no-show (55\%), having a recent prior colonoscopy $(22 \%)$, or that the patient could not be reached (13\%) [21]. Our current study extends these findings by specifying the distribution of reasons for each intermediate step. Specifically, our findings highlight the importance of competing health concerns and being given a second FIT (to repeat the test) as key barriers for patients who did not receive a referral; and of having had a recent colonoscopy, lacking insurance authorization/medical clearance, or having inadequate bowel prep as key barriers for patients with who completed a pre-procedure visit but did not receive a colonoscopy. Notably, these reasons are not always patient-reported, and leave unanswered questions about why patients may have declined or failed to show up for an appointment. Such information could be obtained from direct patient interviews. Moreover, the low proportion of charts with documented reasons for no colonoscopy may underscore the need to promote informed discussions between providers and patients about the risk of late cancer detection among patients who discontinue the screening process [6]. Improving the documentation of reasons for no follow-up could better inform future interventions to improve follow-up care.

The substantial drop-off that occurred between the referral and the pre-procedure visit raises the question of whether the pre-procedure visit creates unnecessary

Table 3 Summary of chart-abstracted reasons for not completing a given step in the screening pathway

\begin{tabular}{|c|c|c|c|c|c|c|c|c|}
\hline \multirow{2}{*}{$\begin{array}{l}\text { Total N with a reason }{ }^{\mathrm{a}} \\
\text { Reasons }\end{array}$} & \multicolumn{2}{|c|}{$\begin{array}{l}\text { No referral } \\
n=69\end{array}$} & \multicolumn{2}{|c|}{$\begin{array}{l}\text { No pre-procedure } \\
\text { visit } \\
n=277\end{array}$} & \multicolumn{2}{|c|}{$\begin{array}{l}\text { No colonoscopy } \\
n=56\end{array}$} & \multicolumn{2}{|c|}{$\begin{array}{l}\text { Overall } \\
\mathrm{N}=402\end{array}$} \\
\hline & $\mathbf{N}$ & $\%$ & $\mathbf{N}$ & $\%$ & $\mathrm{~N}$ & $\%$ & $\mathbf{N}$ & $\%$ \\
\hline Patient declined, no-show, canceled & 13 & 18.8 & 57 & 20.6 & 7 & 12.5 & 77 & 19.2 \\
\hline Provider unable to reach patient & 4 & 5.8 & 53 & 19.1 & 2 & 3.6 & 59 & 14.7 \\
\hline Competing health concerns/ end-of-life/ deceased & 11 & 15.9 & 15 & 5.4 & 5 & 8.9 & 31 & 7.7 \\
\hline Patient barriers (e.g. transportation, insurance, unstable housing) & 3 & 4.3 & 13 & 4.7 & 3 & 5.4 & 19 & 4.7 \\
\hline Patient given a 2nd FIT & 8 & 11.6 & 10 & 3.6 & 0 & 0.0 & 18 & 4.5 \\
\hline Recent colonoscopy & 6 & 8.7 & 2 & 0.7 & 6 & 10.7 & 14 & 3.5 \\
\hline Gl authorization/ clearance not obtained, inadequate prep ${ }^{b}$ & 2 & 2.9 & 5 & 1.8 & 7 & 12.5 & 14 & 3.5 \\
\hline Patient moved/ transferred care & 2 & 2.9 & 7 & 2.5 & 0 & 0.0 & 9 & 2.2 \\
\hline Unknown-no indication in chart & 21 & 30.4 & 111 & 40.1 & 24 & 42.9 & 156 & 38.8 \\
\hline
\end{tabular}

Bolded text indicates top four reasons for each step

${ }^{a} A$ given patient's chart could have more than one reason

${ }^{\mathrm{b}}$ Includes 1 chart noting that the PCP did not follow-up with patient 
burden for patients to attend colonoscopy procedures. Further research is needed to address this question. Given the recent and rapid adoption of telehealth as a replacement for in-person visits during the COVID-19 pandemic, this question could potentially be answered using a natural experiment comparing colonoscopy completion rates among patients attending telehealth preprocedure visits and in-person pre-procedure visits.

Our findings have important implications for the design and adaptation of patient navigation programs and other interventions to address barriers to completing follow-up colonoscopy. First, our findings suggest that efforts are most needed to support referred patients in establishing care with GI practices. These supports are particularly needed for patients aged 65 and older, those who lack health insurance, and those who have not attended a clinic visit in the year prior to an abnormal FIT result. Prediction modeling could be useful to further identify patients who may benefit most from such supports [15]. Moreover, our findings show that while fewer than half of patients with an abnormal FIT completed a colonoscopy, time to colonoscopy completion overall was within the timeframe of national benchmarks, possibly suggesting an absence of prolonged scheduling delays among those who successfully access care.

Innovations in non-invasive screening technology, such as urine tests, exhaled breath tests, and blood-based tests, could minimize patient stress and discomfort and improve adherence to CRC screening [23, 24]. However, research is scarce on the performance of these innovations to identify patients with advanced adenomas [24]. Irrespective of first-line test choice, efforts will likely still be needed to assure that those with limited access to health care can get a follow-up colonoscopy.

\section{Strengths and limitations}

A primary strength of this study is our large retrospective sample from a non-integrated $\mathrm{FQHC}$ that refers patients to several community GI practices. Additionally, our research team conducted complete health record abstraction, and we report findings across multiple steps in the colonoscopy process. Nevertheless, our study has several limitations. While our study relied on chartabstracted data and requests of colonoscopy records from GI practices, we cannot be sure that all records of completed colonoscopies were in the EHR; thus, our reported colonoscopy completion rates may underestimate true rates. However, given the high concordance between EHR-query-obtained and chart-abstracted results (92\% for GI referral; 96\% for colonoscopy completion), we can be reasonably confident our data include nearly all procedures. Our analysis was also limited to information in the health record, which inherently omits important system-level factors, such as referral tracking procedures, protocols to follow up with patients, health record interoperability, and available staffing resources [25]. Moreover, reasons for non-completion recorded in the EHR are incomplete and we did not gather qualitative data directly from patients who did not complete the recommended colonoscopy. Finally, our findings are from a single FQHC in Western Washington and may not generalize to other settings.

\section{Conclusions}

Despite the elevated risk of CRC among individuals with abnormal FIT results, rates of follow-up colonoscopy in a non-integrated FQHC were substantially below the $80 \%$ target set by the Multi-Society Task Force on Colorectal Cancer. Efforts to improve rates of follow-up colonoscopy might focus on supporting the care transition between primary to specialty GI care, and emphasizing care for patients aged 65-75 years, those without a recent clinic visit, and those who lack health insurance.

\section{Abbreviations}

CRC: Colorectal cancer; FIT: Fecal immunochemical test; FQHC: Federally qualified health center; EHR: Electronic health record; GI: Gastroenterology.

\section{Acknowledgements}

Not applicable.

Authors' contributions

GDC, AFP conceptualized the study and GDC drafted the manuscript. AKS gathered the data; MTS and JSR analyzed the data; JHT managed the project; RJ, JG, NY, and RRM provided clinical oversight for the study. All authors have read and approved the final manuscript.

\section{Funding}

Research reported in this publication was supported by the National Cancer Institute of the National Institutes of Health under Award Number R01CA218923. The content is solely the responsibility of the authors and does not necessarily represent the official views of the National Institutes of Health. The National Institutes of Health had no role in the study design; the collection, analysis and interpretation of data; the writing of the report; or the decision to submit the article for publication.

\section{Availability of data and materials}

The datasets analyzed during the current study and the full trial protocol are available from the corresponding author on reasonable request.

\section{Declarations}

\section{Ethics approval and consent to participate}

The study was reviewed by the Kaiser Permanente Northwest Institutional Review Board [protocol \# STUDY00000779]; the study was granted a waiver of informed consent because it posed minimal risks to patients. Thus, no written nor verbal consent was obtained.

\section{Consent for publication}

Not applicable.

\section{Competing interests}

From September 2017-June 2018, GDC served as the Principal Investigator on an industry funded study (Quidel Corporation) to compare the clinical 
performance of an experimental FIT to an FDA-approved FIT. In 2020-2021, GDC served as a scientific advisor for Exact Sciences and Guardant Health. All other authors declare no conflicts of interest.

\section{Author details}

${ }^{1}$ Center for Health Research, Kaiser Permanente Northwest, 3800 North Interstate Avenue, Portland, OR 97227, USA. ${ }^{2}$ Oregon Health Sciences University, Portland, OR, USA. ${ }^{3}$ Sea Mar Community Health Centers, Seattle, WA, USA.

${ }^{4}$ Northwest Permanente Medical Group, Portland, OR, USA.

Received: 14 July 2021 Accepted: 14 September 2021

Published online: 28 September 2021

\section{References}

1. American Cancer Society. Cancer Facts \& Figures 2019. Atlanta, GA. 2019.

2. Levin TR, Corley DA, Jensen CD, et al. Effects of organized colorectal cancer screening on cancer incidence and mortality in a large communitybased population. Gastroenterology. 2018;155(5):1383-1391.e1385.

3. Bharti B, May FFP, Nodora J, et al. Diagnostic colonoscopy completion after abnormal fecal immunochemical testing and quality of tests used at 8 federally qualified health centers in Southern California: opportunities for improving screening outcomes. Cancer. 2019:125:4203-9.

4. Liss DT, Brown T, Lee JY, et al. Diagnostic colonoscopy following a positive fecal occult blood test in community health center patients. Cancer Causes Control. 2016;27(7):881-7.

5. Coronado GD, Petrik AF, Vollmer WM, et al. Effectiveness of a mailed colorectal cancer screening outreach program in community health clinics: The STOP CRC cluster randomized clinical trial. JAMA Intern Med. 2018:178(9):1174-81.

6. Issaka RB, Singh MH, Oshima SM, et al. Inadequate utilization of diagnostic colonoscopy following abnormal fit results in an integrated safety-net system. Am J Gastroenterol. 2017;112(2):375-82.

7. Thamarasseril S, Bhuket T, Chan C, Liu B, Wong RJ. The need for an integrated patient navigation pathway to improve access to colonoscopy after positive fecal immunochemical testing: a safety-net hospital experience. J Community Health. 2017;42(3):551-7. https://doi.org/10.1007/ s10900-10016-10287-10902.

8. Robertson DJ, Lee JK, Boland CR, et al. Recommendations on fecal immunochemical testing to screen for colorectal neoplasia: a consensus statement by the US Multi-Society Task Force on colorectal cancer. Gastrointest Endosc. 2017;85(1):2-21.e23.

9. Lee YC, Fann JC, Chiang TH, et al. Time to colonoscopy and risk of colorectal cancer in patients with positive results from fecal immunochemical tests. Clin Gastroenterol Hepatol. 2019:17(7):1332-1340.e1333.

10. Corley DA, Jensen CD, Quinn VP, et al. Association between time to colonoscopy after a positive fecal test result and risk of colorectal cancer and cancer stage at diagnosis. JAMA. 2017;317(16):1631-41.

11. Meester RG, Zauber AG, Doubeni CA, et al. Consequences of increasing time to colonoscopy examination after positive result from fecal colorectal cancer screening test. Clin Gastroenterol Hepatol. 2016;14(10):1445-1451.e1448.

12. Jones RM, Woolf SH, Cunningham TD, et al. The relative importance of patient-reported barriers to colorectal cancer screening. Am J Prev Med. 2010;38(5):499-507

13. Quick BW, Hester CM, Young KL, Greiner KA. Self-reported barriers to colorectal cancer screening in a racially diverse, low-income study population. J Community Health. 2013;38(2):285-92.

14. O'Malley AS, Beaton E, Yabroff KR, Abramson R, Mandelblatt J. Patient and provider barriers to colorectal cancer screening in the primary care safety-net. Prev Med. 2004;39(1):56-63.

15. Coronado GD, Johnson ES, Leo MC, et al. Patient randomized trial of a targeted navigation program to improve rates of follow-up colonoscopy in community health centers. Contemp Clin Trials. 2019:89:105920.

16. Lee YC, Li-Sheng Chen S, Ming-Fang Yen A, et al. Association between colorectal cancer mortality and gradient fecal hemoglobin concentration in colonoscopy noncompliers. J Natl Cancer Inst. 2017;109(5):djw269.

17. Imperiale TF, Ransohoff DF, Itzkowitz SH, et al. Multitarget stool DNA testing for colorectal-cancer screening. N Engl J Med. 2014;370(14):1287-97.

18. Heitman SJ, Ronksley PE, Hilsden RJ, Manns BJ, Rostom A, Hemmelgarn BR. Prevalence of adenomas and colorectal cancer in average risk individuals: a systematic review and meta-analysis. Clin Gastroenterol Hepatol. 2009;7(12):1272-8.

19. Knudsen $A B$, Zauber $A G$, Rutter $C M$, et al. Estimation of benefits, burden, and harms of colorectal cancer screening strategies: modeling study for the US Preventive Services Task Force. JAMA. 2016;315(23):2595-609.

20. Chubak J, Garcia MP, Burnett-Hartman AN, et al. Time to colonoscopy after positive fecal blood test in four U.S. health care systems. Cancer Epidemiol Biomarkers Prev. 2016;25(2):344-50.

21. O'Connor EA, Nielson CM, Petrik AF, Green BB, Coronado GD. Prospective cohort study of predictors of follow-up diagnostic colonoscopy from a pragmatic trial of FIT screening. Sci Rep. 2020;10(1):2441. https://doi.org/ 10.1038/s41598-41020-59032-41590.

22. Doubeni CA, Gabler NB, Wheeler CM, et al. Timely follow-up of positive cancer screening results: a systematic review and recommendations from the PROSPR Consortium. CA Cancer J Clin. 2018;68(3):199-216.

23. Altomare DF, Picciariello A, Rotelli MT, et al. Chemical signature of colorectal cancer: case-control study for profiling the breath print. BJS Open. 2020.

24. Ferrari A, Neefs I, Hoeck S, Peeters M, Van Hal G. Towards novel noninvasive colorectal cancer screening methods: a comprehensive review. Cancers (Basel). 2021:13(8):1820.

25. Sharma AE, Lyson HC, Cherian R, Somsouk M, Schillinger D, Sarkar U. A root cause analysis of barriers to timely colonoscopy in california safetynet health systems. J Patient Saf. 2020. https://doi.org/10.1097/PTS.00000 00000000718.

\section{Publisher's Note}

Springer Nature remains neutral with regard to jurisdictional claims in published maps and institutional affiliations.

\footnotetext{
Ready to submit your research? Choose BMC and benefit from:

- fast, convenient online submission

- thorough peer review by experienced researchers in your field

- rapid publication on acceptance

- support for research data, including large and complex data types

- gold Open Access which fosters wider collaboration and increased citations

- maximum visibility for your research: over $100 \mathrm{M}$ website views per year
}

At BMC, research is always in progress.

Learn more biomedcentral.com/submissions 